\title{
Cross recurrence plot based synchronization of time series
}

\author{
N. $\operatorname{Marwan}^{1}$, M. Thiel ${ }^{1}$, and N. R. Nowaczyk ${ }^{2}$ \\ ${ }^{1}$ Institute of Physics, University of Potsdam, Germany \\ ${ }^{2}$ GeoForschungs-Zentrum Potsdam, Germany
}

Received: 3 September 2001 - Accepted: 25 October 2001

\begin{abstract}
The method of recurrence plots is extended to the cross recurrence plots (CRP) which, among others, enables the study of synchronization or time differences in two time series. This is emphasized in a distorted main diagonal in the cross recurrence plot, the line of synchronization (LOS). A non-parametrical fit of this LOS can be used to rescale the time axis of the two data series (whereby one of them is compressed or stretched) so that they are synchronized. An application of this method to geophysical sediment core data illustrates its suitability for real data. The rock magnetic data of two different sediment cores from the Makarov Basin can be adjusted to each other by using this method, so that they are comparable.
\end{abstract}

\section{Introduction}

The adjustment of data sets with various time scales occurs on many occasions, e.g. data preparation of tree rings or geophysical profiles. In geology, often a large set of geophysical data series is taken at various locations (e.g. sediment cores). That is why these data series have a different length and time scale. Before any time series analysis can be started, the data series have to be synchronized to the same time scale. Usually, this is done visually by comparing and correlating each maximum and minimum in both data sets by hand (wiggle matching), which includes the human factor of subjectiveness and is a lengthy process. An automatic and objective method for verification should be very welcome.

In the last decades some techniques for this kind of correlation and adjustment were suggested. They span graphical methods (Prell et al., 1986), inverse algorithms, e.g. using Fourier series (Martinson et al., 1982) and algorithms based on similarity of data, e.g. sequence slotting (Thompson and Clark, 1989).

However, we focus on a method based on nonlinear time series analysis. During our investigations of the method of

Correspondence to: N. Marwan

(marwan@agnld.uni-potsdam.de) cross recurrence plots (CRP), we have found an interesting feature. Besides the possibility of application of the recurrence quantification analysis (RQA) of Webber and Zbilut on CRPs (1994), there is a more fundamental relation between the structures in the CRP and the considered systems. This feature can be used for synchronization of data sets. Although the first steps of this method are similar to the sequence slotting method, their roots are different.

First we give an introduction to CRPs. Then we explain the relationship between the structures in the CRP and the systems and illustrate this with a simple model. Finally, we apply the CRP to geophysical data in order to synchronize various profiles and to show their practical availability. Since we focus on the synchronization feature of the CRP, we will not give a comparison between the different alignment methods.

\section{The Recurrence Plot}

Recurrence plots (RP) were firstly introduced by Eckmann et al. (1987) in order to visualize time dependent behaviour of orbits $\boldsymbol{x}_{i}$ in phase space. An RP represents the recurrence of the phase space trajectory to a state. The recurrence of states is a fundamental property of deterministic dynamical systems (Argyris et al., 1994; Casdagli, 1997; Kantz and Schreiber, 1997). The main step in the visualization is the calculation of the $N \times N$-matrix

$\mathbf{R}_{i, j}=\Theta\left(\varepsilon-\left\|\boldsymbol{x}_{i}-\boldsymbol{x}_{j}\right\|\right), \quad i, j=1 \ldots N$,

where $\varepsilon$ is a predefined cutoff distance, $\|\cdot\|$ is the norm (e.g. the Euclidean norm) and $\Theta(x)$ is the Heaviside function. The values one and zero in this matrix can be simply visualized by the colours black and white. Depending on the kind of application, $\varepsilon$ can be a fixed value or it can be changed for each $i$ in such a way that in the ball with the radius $\varepsilon$ a predefined amount of neighbours occurs. The latter will provide a constant density of recurrence points in each column of the RP.

The recurrence plot exhibits characteristic patterns for typical dynamical behaviour (Eckmann et al., 1987; Webber Jr. 
and Zbilut, 1994): A collection of single recurrence points, homogeneously and irregularly distributed over the whole plot, reveals a mainly stochastic process. Longer parallel diagonals, formed by recurrence points and with the same distance between the diagonals, are caused by periodic processes. A paling of the RP away from the main diagonal to the corners reveals a drift in the amplitude of the system. Vertical and horizontal white bands in the RP result from states which occur rarely or represent extremes. Extended horizontal and vertical black lines or areas occur if a state does not change for some time, e.g. laminar states. All these structures were formed by using the property of recurrence of states. It should be pointed out that the states are only the "same" and recur in the sense of the vicinity, which is determined by the distance $\varepsilon$. RPs and their quantitative analysis (RQA) became better known in the last decade (e.g. Casdagli, 1997). Their applications to a wide field of miscellaneous research show their suitability in the analysis of short and non-stationary data.

\section{The Cross Recurrence Plot}

Analogous to Zbilut et al. (1998), we have expanded the method of recurrence plots (RP) to the method of cross recurrence plots. In contrast to the conventional RP, two time series are simultaneously embedded in the same phase space. The test for closeness of each point of the first trajectory $x_{i}$ $(i=1 \ldots N)$ with each point of the second trajectory $y_{j}$ $(j=1 \ldots M)$ results in a $N \times M$ array

$\mathbf{C R}_{i, j}=\Theta\left(\varepsilon-\left\|\boldsymbol{x}_{i}-\boldsymbol{y}_{j}\right\|\right)$.

The visualization of this is called the cross recurrence plot. The definition of the closeness between both trajectories can be varied as described above. Varying $\varepsilon$ may be useful to handle systems with different amplitudes.

The CRP compares the considered systems and allows us to benchmark the similarity of states. In this paper, we focus on the bowed "main diagonal" in the CRP, because it is related to the frequencies and phases of the systems considered.

\section{The line of synchronization in the CRP}

Regarding the conventional RP, Eq. (1), one always finds a main diagonal in the plot because the $(i, i)$-states are identical. The RP can be considered as a special case of the CRP, Eq. (2), which usually does not have a main diagonal as the $(i, i)$-states are not identical.

In data analysis one is often faced with time series that are measured on varying time scales. These could be sets from borehole or core data in geophysics or tree rings in dendrochronology. Sediment cores might have undergone a number of coring disturbances such as compression or stretching. Moreover, cores from different sites with differing sedimentation rates would have different temporal reso-

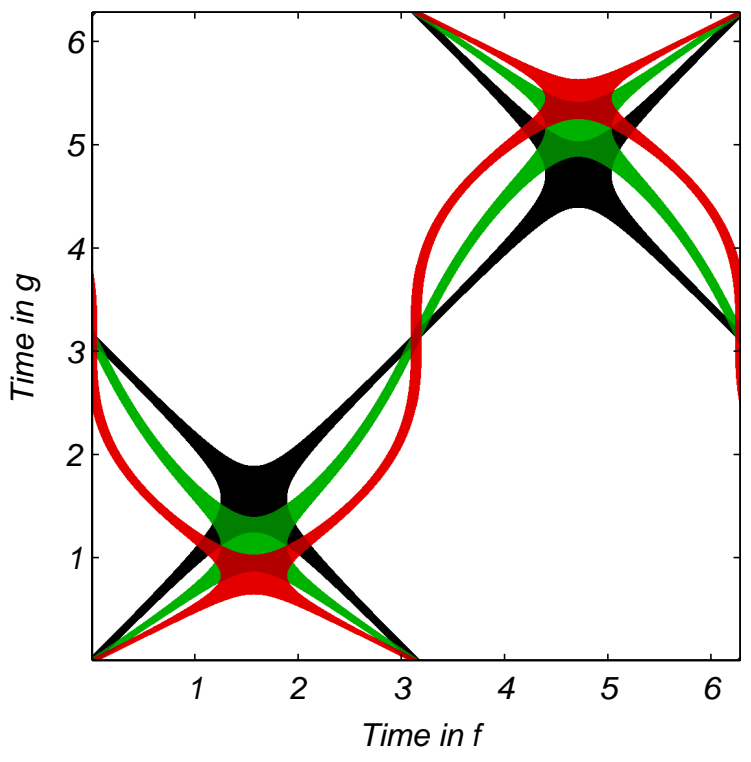

Fig. 1. Cross recurrence plots of sine functions $f(t)=\sin (\varphi t)$ and $g(t)=\sin (\varphi t+a \sin (\psi t))$, where $a=0$ for the black CRP, $a=0.5$ for the green CRP and $a=1$ for the red CRP. The variation in the time domain leads to a deforming of the synchronization line.

lutions. All these factors require a method of synchronization.

A CRP of two corresponding time series will not contain a main diagonal. But, if the sets of data are similar, e.g. only rescaled, a more or less continuous line in the CRP that is like a distorted main diagonal can occur. This line contains information on the rescaling. We give an illustrative example. A CRP of a sine function with itself (i.e. this is the RP) contains a main diagonal (black CRP in Fig. 1). Hence, the CRPs in the Fig. 1 are computed with embeddings of dimension one; further diagonal lines from the upper left to the lower right occur. These lines typify the similarity of the phase space trajectories in positive and negative time direction.

Now we rescale the time axis of the second sine function in the following way

$\sin (\varphi t) \longrightarrow \sin (\varphi t+a \sin (\psi t))$

We will henceforth use the notion rescaling only in the mention of the rescaling of the time scale. The rescaling of the second sine function, with different parameters $\varphi$, results in a deformation of the main diagonal (green and red CRP in Fig. 1). The distorted line contains the information on the rescaling which we will need in order to re-synchronize the two time series. Therefore, we call this distorted diagonal the line of synchronization (LOS).

In the following, we present a toy function to explain the procedure. If we consider a one dimensional case without embedding, the CRP is computed with

$\mathbf{C R}\left(t_{1}, t_{2}\right)=\Theta\left(\varepsilon-\left\|f\left(t_{1}\right)-g\left(t_{2}\right)\right\|\right)$. 


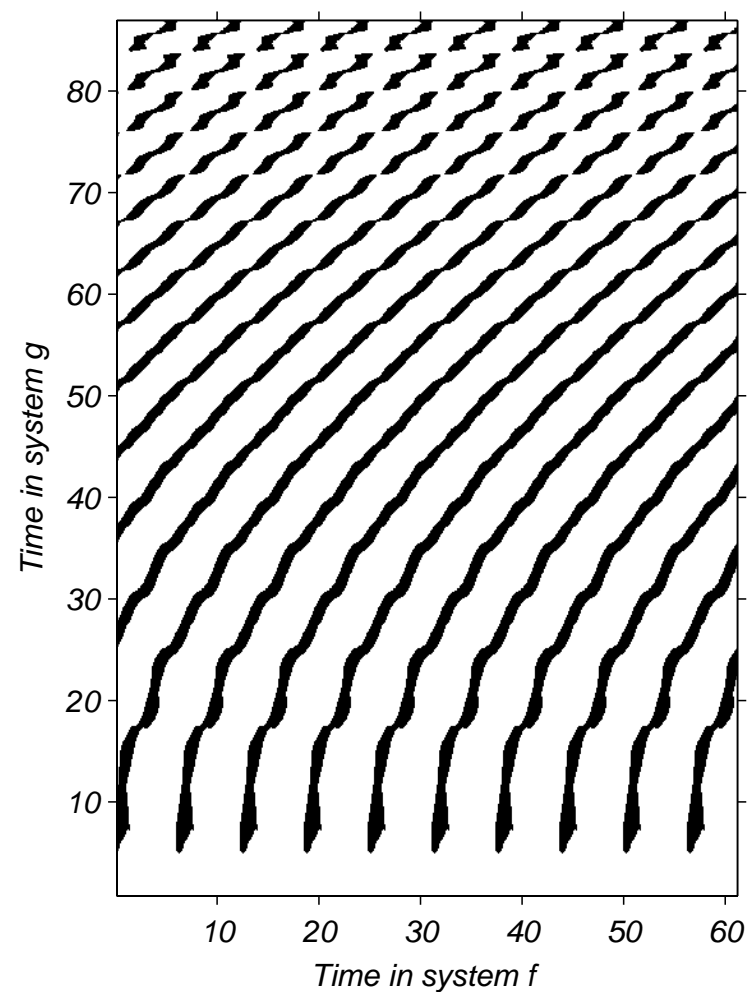

Fig. 2. Cross recurrence plots of two sine functions $f(t)=\sin (\varphi t)$ and $\left.g(t)=\sin \left(\psi t^{2}\right)\right)$ which is the basis of the determination of the rescaling function between both data series. The embedding parameters were dimension $m=2$, delay $\tau=\pi / 2$ and a varying threshold $\varepsilon$, in such a way that the CRP contains a constant recurrence density of $20 \%$.

If we set $\varepsilon=0$ to simplify the condition, Eq. (4) gives a recurrence point if

$f\left(t_{1}\right)=g\left(t_{2}\right)$.

In general, this is an implicit condition that links the variable $t_{1}$ to $t_{2}$. Considering physical examples of above, it can be assumed that the time series are essentially the same - this means that $f=g-$ up to a rescaling function of time. So we can state that

$f\left(t_{1}\right)=f\left(\phi\left(t_{1}\right)\right)$.

If the functions $f(\cdot)$ and $g(\cdot)$ are not identical, our method is, in general, not capable of deciding if the difference in the time series is due to different dynamics $(f(\cdot) \neq g(\cdot))$ or if it is due to simple rescaling. So the assumption that the dynamics are alike up to a rescaling in time is essential, even though, for some cases where $f \neq g$, it can be applied in the same way. If we consider the functions $f(\cdot)=a \cdot \bar{f}(\cdot)+b$ and $g(\cdot)=\bar{g}(\cdot)$, where $f(\cdot) \neq g(\cdot)$ are the observations and $\bar{f}(\cdot)=\bar{g}(\cdot)$ are the states, normalization with respect to the mean and the standard deviation allows us to use our method.

$f(\cdot)=a \cdot \bar{f}(\cdot)+b \longrightarrow \tilde{f}(\cdot)=\frac{f(\cdot)-\langle f(\cdot)\rangle}{\sigma(f(\cdot))}$

$\tilde{g}(\cdot)=\frac{g(\cdot)-\langle g(\cdot)\rangle}{\sigma(g(\cdot))}$

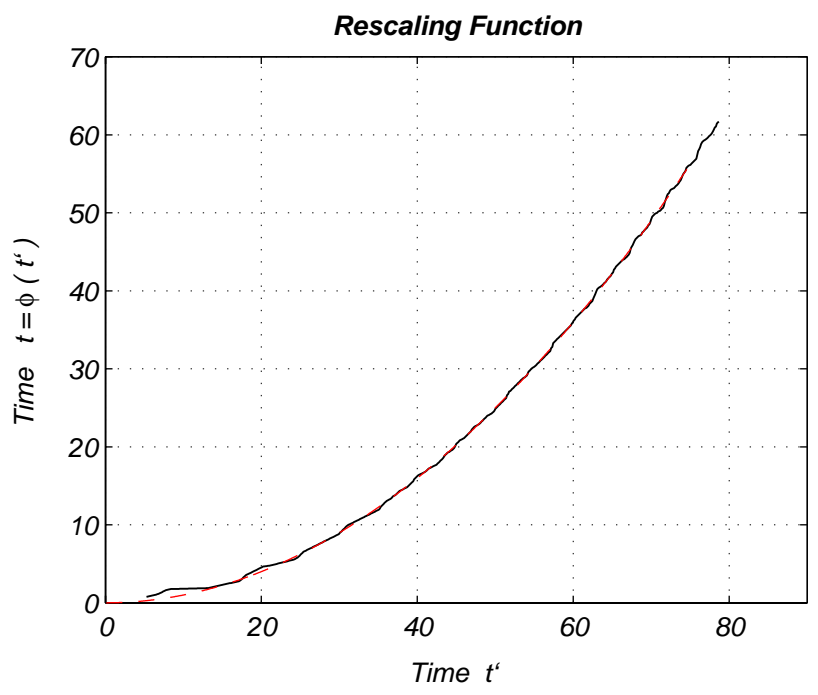

Fig. 3. The rescaling function (black) determined from the CRP in Fig. 2. This has the expected parabolic shape of the squared coherence in the time domain. In red the square function.

With $\bar{g}(\cdot)=\bar{f}(\cdot)$ the functions $\tilde{f}(\cdot)$ and $\tilde{g}(\cdot)$ are the same after the normalization. Then our method can be applied without any further modification.

In some special cases Eq. (6) can be resolved with respect to $t_{1}$. Such a case is a system of two sine functions with different frequencies

$$
\begin{aligned}
& f(t)=\sin (\varphi \cdot t+\alpha) \\
& g(t)=\sin (\psi \cdot t+\beta)
\end{aligned}
$$

Using Eq. (5) and Eq. (6) we find

$$
\sin \left(\varphi t_{1}+\alpha\right)-\sin \left(\psi t_{2}+\beta\right)=0
$$

and one explicit solution of this equation is

$$
\Rightarrow \quad t_{2}=\phi\left(t_{1}\right)=\left(\frac{\varphi}{\psi} t_{1}+\gamma\right)
$$

with $\gamma=(\alpha-\beta) / \psi$. In this special case the slope of the main line in a cross recurrence plot represents the frequency ratio and the distance between the axes origin and the intersection of the line of synchronization with the ordinate gives the phase difference. The function $t_{2}=\phi\left(t_{1}\right)$ (Eq. 6) is a transfer or rescaling function which allows us to rescale the second system to the first system. If the rescaling function is not linear the LOS will also be curved.

For the application, one has to determine the LOS - usually non-parametrically - and then rescale one of the time series. In the Appendix we describe a simple algorithm for estimating the LOS. Its determination will be better for higher embeddings because the vertical and cross-diagonal structures will vanish. Note that the embedding of the time series involves difficulties. The Takens Embedding Theorem holds for closed, deterministic systems without noise only. If noise is present, one needs its realization to find a reasonable embedding. For stochastic time series it does not make sense 

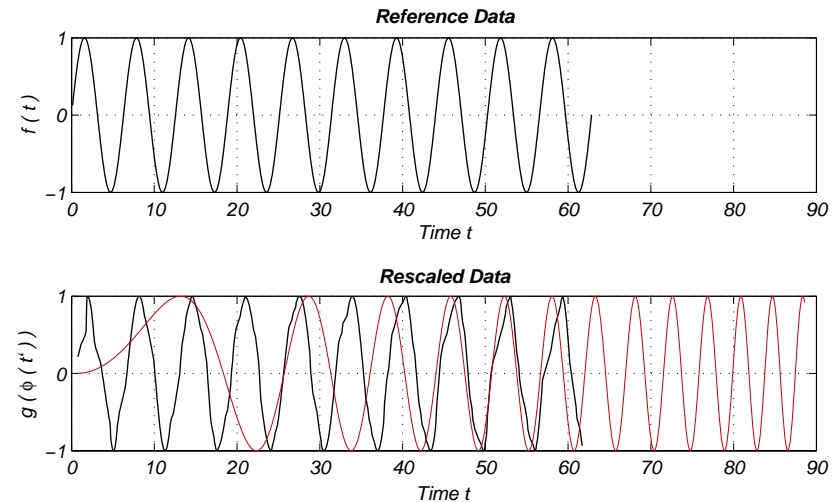

Fig. 4. Reference data series (upper panel) and rescaled data series before (red) and after (black) using the rescaling function of Fig. 3 (lower panel).

to consider a phase space and so embedding is, in general, not justified here either (Romano, to be published; Takens, 1981).

The choice of a special embedding lag could be correct for one section of the data but incorrect for another (for an example see below). This can be the case if the data is nonstationary. Furthermore, the choice of method for computing the CRP and the threshold $\varepsilon$ will influence the quality of the estimated LOS.

The next sections will be dedicated to application.

\section{Application to a simple example}

First, we consider two sine functions, $f(t)=\sin (\varphi t)$ and $g(t)=\sin \left(\psi t^{2}\right)$, where the time scale of the second sine differs from the first by a quadratic term and the frequency $\psi=0.01 \varphi$. Sediment parameters are related to such kind of functions because gravity pressure increases nonlinearly with the depth. It can be assumed that both data series come from the same process and were subjected to different deposital compressions (e.g. a squared or exponential increasing of the compression). Their CRP contains a bowed LOS (Fig. 2). We have used the embedding parameters dimension $m=2$, delay $\tau=\pi / 2$ and a varying threshold $\varepsilon$, so that the CRP contains a constant recurrence density of $20 \%$. Assuming that the time scale of $g$ is not the correct scale, we denote that scale by $t^{\prime \prime}$. In order to determine the non-parametrical LOS, we have implemented the algorithm described in the Appendix. Although this algorithm is still not mature, we obtained reliable results (Fig. 3). The resulting rescaling function has the expected squared shape $t=\phi\left(t^{\prime \prime}\right)=0.01 t^{\prime \prime 2}$ (red curve in Fig. 3). Substituting the time scale $t^{\prime \prime}$ in the second data series $g\left(t^{\prime \prime}\right)$ by this rescaling function $t=\phi\left(t^{\prime \prime}\right)$, we get a set of synchronized data $f(t)$ and $g(t)$ with the nonparametric rescaling function $t=\phi\left(t^{\prime \prime}\right)$ (Fig. 4). The synchronized data series are approximately the same. The cause of some differences is the meandering of the LOS which itself is caused by partial weak embedding. Nevertheless, this

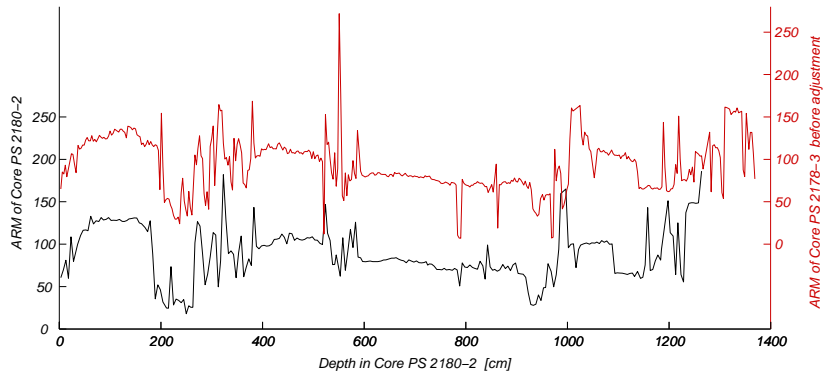

Fig. 5. ARM data of the boreholes PS 2178-3 GPC and PS 21802 GPC in the Central Arctic Ocean before adjustment.

can be avoided by using a more complex algorithm for estimating the LOS.

\section{Application to real data}

In order to continue the illustration of the working of our method we have applied it to real data from geology.

In the following, we compare the method of cross recurrence plot matching with the conventional method of visual wiggle matching (interactive adjustment). Geophysical data of two sediment cores from the Makarov Basin, central Arctic Ocean, PS 2178-3 and PS 2180-2, were analysed. The task should be to adjust the data of the PS 2178-3 data (data length $N=436$ ) to the scale of the PS 2180-2 (data length $N=251$ ) in order to get a depth-depth-function which allows us to synchronize both data sets (Fig. 5).

We have constructed the phase space with six normalized parameters, low field magnetic susceptibility $\left(\kappa_{L F}\right)$, anhysteretic remanent magnetization $(A R M)$, ratio of anhysteretic susceptibility to $\kappa_{L F}\left(\kappa_{A R M} / \kappa_{L F}\right)$, relative palaeointensity $(P J A)$, median destructive field of $A R M\left(M D F_{A R M}\right)$ and inclination $(I N C)$. A comprehensive discussion of the data is given in Nowaczyk et al. (2001). The embedding was combined with the time-delayed method according to Takens (1981) in order to increase further the dimension of the phase space with the following rule: If we have $n$ parameters $a_{i}$, the embedding with dimension $m$ and delay $\tau$ will result in a $(m \cdot n)$-dimensional phase space:

$$
\begin{aligned}
\boldsymbol{x}(t)= & \left(a_{1}(t), \ldots, a_{n}(t),\right. \\
& a_{1}(t+\tau), \ldots, a_{n}(t+\tau), \\
& a_{1}(t+2 \tau), \ldots, a_{n}(t+2 \tau), \ldots \\
& a_{1}(t+(m-1) \tau), \ldots, a_{n}(t+(m-1) \tau)
\end{aligned}
$$

For our investigation we have used a dimension $m=3$ and a delay $\tau=1$, which finally led to a phase space of dimension $18(3 \times 6)$. The recurrence criterion was $\varepsilon=5 \%$ nearest neighbours.

The resulting CRP shows a clear LOS and some clustering of black patches (Fig. 6). The latter occurs due to the plateaux in the data. The next step is to fit a non-parametric 


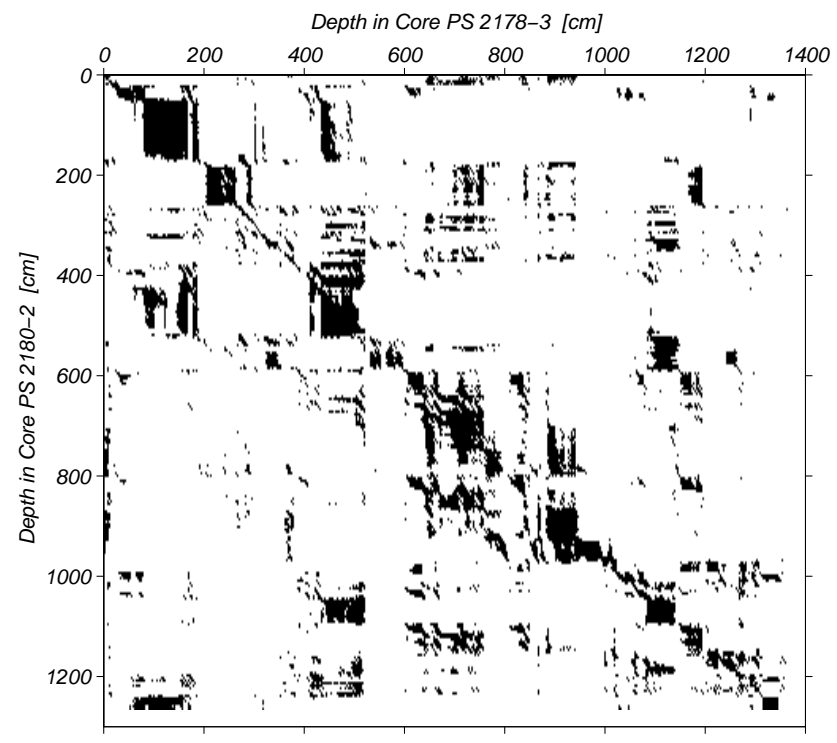

Fig. 6. Cross recurrence plot based on six normalized sediment parameters and an additional embedding dimension of $m=3(\tau=1, \varepsilon=0.05)$.

function (the depth-depth-curve) to the LOS in the CRP (red curve in Fig. 6). With this function we are able to adjust the data of the PS 2178-3 core to the scale of PS 2180-2 (Fig. 8).

The determination of the depth-depth-function with the conventional method of visual wiggle matching is based on interactive and parallel searching for the same structures in the different parameters of both data sets. If the adjustment does not work in a section of the one parameter, one can use another parameter for this section which allows the multivariate adjustment of the data sets. The recognition of the same structures in the data sets requires a degree of experience. However, human eyes are usually better in the visual assessment of complex structures than a computational algorithm.

Our depth-depth-curve differs slightly from the curve which was gained by the visual wiggle matching (Fig. 7). However, despite our (still) weak algorithm used to fit the non-parametric adjustment function to the LOS, we obtained a good result of adjusted data series. If they are well adjusted, the correlation coefficient between the parameters of the adjusted data and the reference data should not vary so much. The correlation coefficients between the reference and adjusted data series is about $0.70-0.80$, where the correlation coefficients of the interactive rescaled data varies from 0.71 -0.87 (Table 1). The $\chi^{2}$ measure of the correlation coefficients emphasizes more variation for the wiggle matching than for the CRP rescaling.

\section{Discussion}

Cross recurrence plots (CRP) reveal similarities in the states of the two systems. A similar trajectory evolution gives a diagonal structure in the CRP. An additional time dilatation

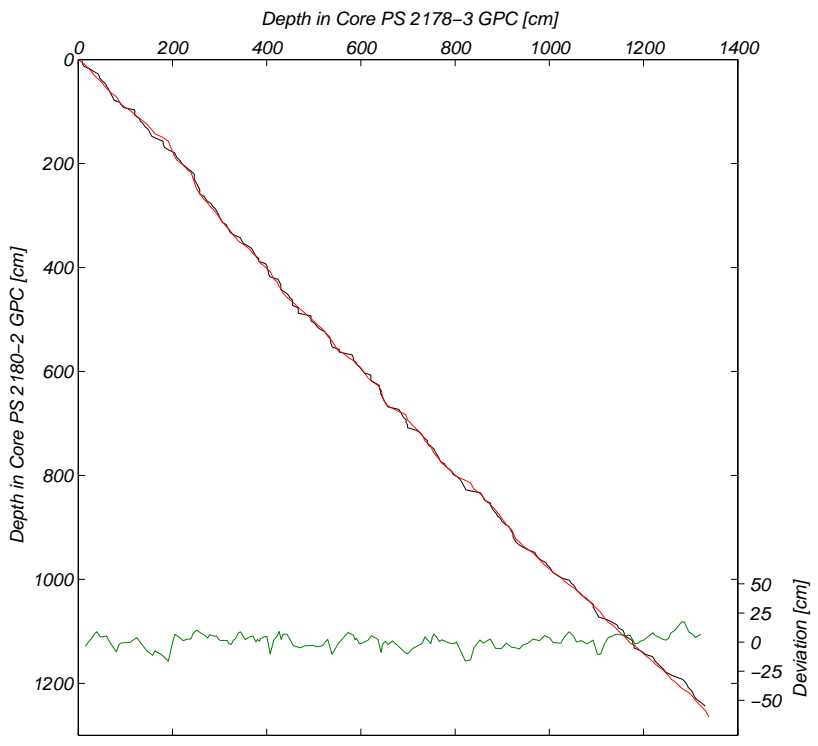

Fig. 7. Depth-depth-curves. In black, the curve gained with the CRP; in red the manually matching result. The green curve shows the deviation between both results.

or compression of one of these similar trajectories causes a distortion of this diagonal structure (Fig. 1). This effect is used to look into the synchronization between both systems. Synchronized systems have diagonal structures along and in the direction of the main diagonal in the CRP. Interruptions of these structures with gaps are possible because of variations in the amplitudes of both systems. However, a loss of synchronization is viewable by the distortion of this structures along the main diagonal (LOS). Fitting a nonparametric function to the LOS allows us to re-synchronize or adjust both systems on the same time scale. Although this method is based on principles from deterministic dynamics, no assumptions about the underlying systems have to be made in order for the method to work.

The first example shows the obvious relationship between the LOS and the time domains of the considered time series. The increasing frequency squared of the second harmonic function causes a parabolic LOS shape in the CRP (Fig. 2). Finally, with this LOS we are able to rescale the second function to the scale of the first harmonic function (Fig. 4). Some differences in the amplitude of the result are caused by the algorithm used in order to extract the LOS from the CRP. However, our concern is to focus on the distorted main diagonal and its relationship with the time domains.

The second example deals with real geological data and allows a comparison with the result of the conventional method of visual wiggle matching. The visual comparison of the adjusted data shows a good concordance with the reference and the wiggle matched data (Fig. 8 and 9). The depth-depthfunction differs up to 20 centimeters from the depth-depthfunction of the wiggle matching. The correlation coefficients between the CRP adjusted data and the reference data varies less than the correlation coefficients of the wiggle matching. 
Table 1. Correlation coefficients $\varrho_{1,2}$ between adjusted data and reference data and their $\chi^{2}$ deviation. The correlation of the interactive adjusted data varies more than the automatic adjusted data. The data length is $N=170$ (wiggle matching) and $N=250$ (CRP matching). The difference between both correlation coefficients $Q_{1}$ and $\varrho_{2}$ is significant at a $99 \%$ significance level when the test measure $\hat{z}$ is greater than $z_{0.01}=2.576$

\begin{tabular}{lrrr}
\hline Parameter & $\varrho_{1}$, wiggle matching & $\varrho_{2}$, CRP matching & $\hat{z}$ \\
\hline$A R M$ & 0.8667 & 0.7846 & 6.032 \\
$M D F_{A R M}$ & 0.8566 & 0.7902 & 4.791 \\
$\kappa_{L F}$ & 0.7335 & 0.7826 & 2.661 \\
$\kappa_{A R M} / \kappa_{L F}$ & 0.8141 & 0.8049 & 0.614 \\
$P J A$ & 0.7142 & 0.6995 & 0.675 \\
$I N C$ & 0.7627 & 0.7966 & 1.990 \\
\hline$\chi^{2}$ & 141.4 & 49.1 & \\
\hline
\end{tabular}

However, the correlation coefficients for the CRP adjusted data are smaller than these for the wiggle matched data. Although their correlation is better, it seems that the interactive method does not produce a balanced adjusting whereas the automatic matching looks for a better balanced adjusting.

These two examples exhibit the ability to work with smooth and non-smooth data whereby the result will be better for smooth data. Small fluctuations in the non-smooth data can be handled by the LOS searching algorithm. Therefore, smoothing strategies, like smoothing or parametrical fit of the LOS, are not necessary. The latter would damp one advantage of this method, that the LOS is yielded as a nonparametrical function. A future task will be the optimization of the LOS searching algorithm in order to get a clear LOS even if the data are non-smooth. Further, the influence of dynamical noise to the result will be studied. Probably, this problem may be bypassed by a suitable LOS searching algorithm too.

Our method has conspicuous similarities with the method of sequence slotting described by Thompson and Clark (1989). The first step in their method is the calculation of a distance matrix, similar to our Eq. (2), which allows the use of multivariate data sets. Thompson and Clark (1989) referred to the distance measure as dissimilarity; this is used to determine the alignment function in such a way that the sum of the dissimilarities along a path in the distance matrix is minimized. This approach is based on dynamic programming methods which were mainly developed for speech pattern recognition in the 70's (e.g. Sakoe and Chiba, 1978). In contrast, RPs were developed to visualize the phase space behaviour of dynamical systems. Therefore, a threshold was introduced to make recurrent states visible. Involvement of a fixed amount of nearest neighbours in phase space and the possibility to increase the embedding dimensions distinguish this approach from the sequence slotting method.

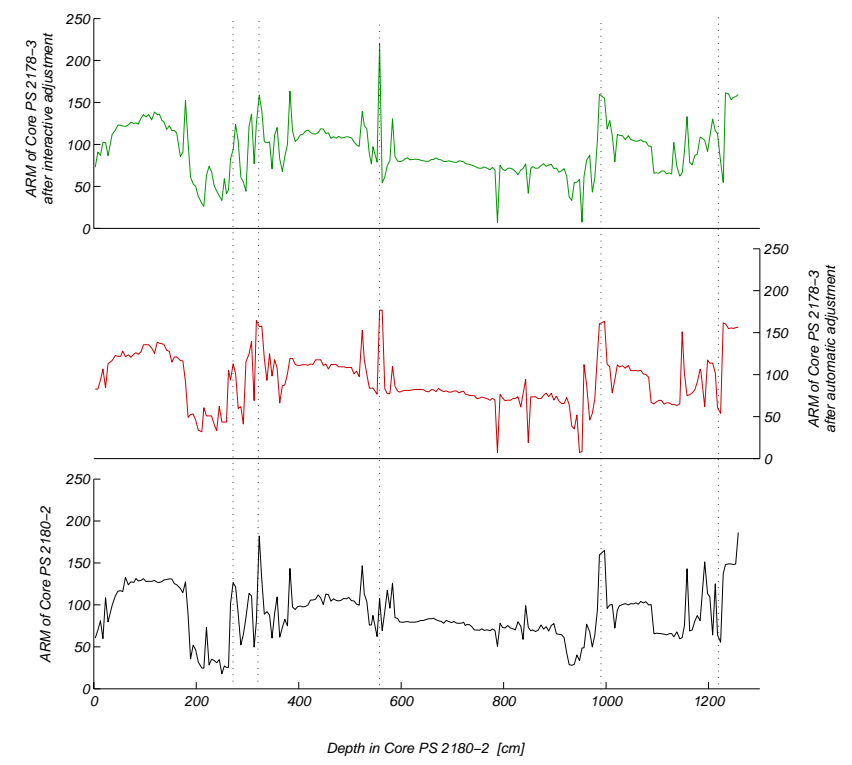

Fig. 8. ARM data after adjustment by wiggle matching (top) and by automatic adjustment using the LOS from Fig. 6. The bottom figure shows the reference data.

\section{Conclusion}

The cross recurrence plot (CRP) can contain information about the synchronization of data series. This is revealed by the distorted main diagonal which is called line of synchronization (LOS). After isolating this LOS from the CRP one obtains a non-parametric rescaling function. With this function one can synchronize the time series. The underlying more-dimensional phase space allows us to include more than one parameter in this synchronization method as it usually appears in geological applications, e.g. core synchronization. The comparison of CRP adjusted geophysical core data with conventional visual matching shows an acceptable reliability level of the new method which can be further improved by a better method for estimating the LOS. The advantage is the automatic, objective and multivariate adjustment. Finally, this method of CRPs can open a wide range of applications as scale adjustment, phase synchronization and pattern recognition, for instance in geology, molecular biology and ecology.

\section{Appendix: An algorithm to fit the LOS}

In order to implement a recognition of the LOS we have used the following simple two-step algorithm. Denote all recurrence points by $r_{i_{\tilde{\alpha}}, j_{\tilde{\beta}}}(\tilde{\alpha}, \tilde{\beta}=1,2, \ldots)$ and the recurrence points lying on the LOS by $r_{i_{\alpha}, j_{\beta}}(\alpha, \beta=1,2, \ldots)$. Before the common algorithm starts, find the recurrence point $r_{i_{1}, j_{1}}$ next to the axes origin. In the first step, the next recurrence point $r_{i_{\tilde{\alpha}}, j_{\tilde{\beta}}}$, after a previous determined recurrence point $r_{i_{\alpha}, j_{\beta}}$, is to be determined. This is carried out by a stepwise increasing of a squared $(w \times w)$ sub-matrix where the 

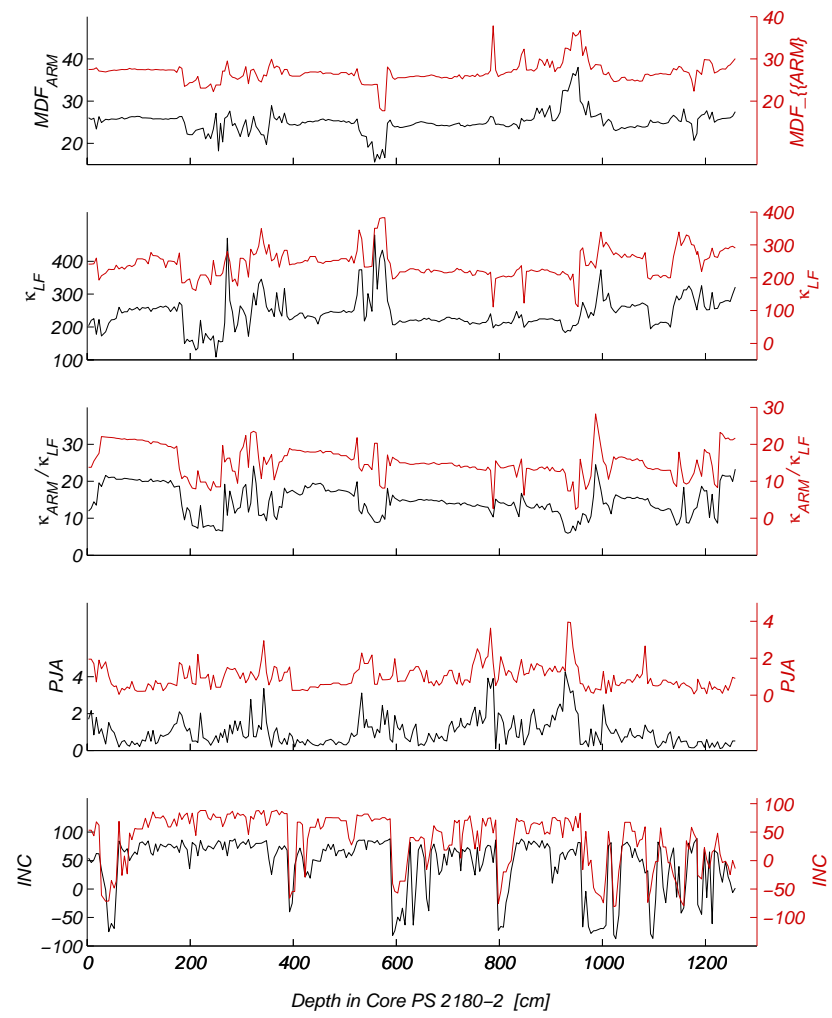

Fig. 9. The adjusted marine sediment parameters. The construction of the CRP was done with the normalized parameters. In these plots we show the parameters, which are not normalized.

previous recurrence point is at the $(1,1)$-location. The size $w$ of this sub-matrix increases step-wise until it meets a new recurrence point or the margin of the CRP. When the next recurrence point $r_{i_{\tilde{\alpha}}, j_{\tilde{\beta}}}=r_{i_{\alpha}+\delta i, j_{\beta}+\delta j}(\delta i=w$ or $\delta j=w)$ in the $x$-direction ( $y$-direction) is found, the second step looks to see if there are following recurrence points in $y$-direction ( $x$-direction). If this is true (e.g. there are a cluster of recurrence points) increase further the sub-matrix in $y$-direction $(x$-direction) until a predefined size $(w+d \tilde{x}) \times(w+d \tilde{y})$ $(d \tilde{x}<d x, d \tilde{y}<d y)$ or until no new recurrence points are met. This further increasing of the sub-matrix is done for the both $x$ - and $y$-direction. Using $d \tilde{x}, d \tilde{y}$ we compute the next recurrence point $r_{i_{\alpha+1}, j_{\beta+1}}$ by determination of the center of mass of the cluster of recurrence points with $i_{\alpha+1}=i_{\alpha}+(d \tilde{x}+\delta i) / 2$ and $j_{\beta+1}=j_{\beta}+(d \tilde{y}+\delta j) / 2$. The latter avoids the fact that the algorithm is driven around widespread areas of recurrence points. Instead of this, the algorithm locates the LOS within these areas. (However, the introducing two additional parameters $d x$ and $d y$ is a disadvantage which should be avoided in further improvements of this algorithm.) The next step is to set the recurrence point $r_{i_{\alpha+1}, j_{\beta+1}}$ to a new start point and to begin with the step one in order to find the next recurrence point. These steps are repeated until the end of the RP is reached.

We know that this algorithm is merely one of many possibilities. The following criteria should be met in order to obtain a good LOS. The number of targeted recurrence points by the LOS $N_{1}$ should converge to a maximum and the number of gaps in the LOS $N_{0}$ should converge to a minimum. An analysis with various estimated LOS confirms this requirement. The correlation between two LOS-synchronized data series arises with $N_{1}$ and with $1 / N_{0}$ (the correlation coefficient correlates most strongly with the ratio $N_{1} / N_{0}$ ).

The algorithm for computation of the CRP and recognition of the LOS are available as Matlab programmes on http://www.agnld.uni-potsdam.de/ marwan.

Acknowledgements. The authors thank J. Kurths and U. Schwarz for continuing support and discussion. This work was supported by the special research programme 1097 of the German Science Foundation (DFG).

\section{References}

Argyris, J. H., Faust, G., and Haase, M.: An Exploration of Chaos, North Holland, Amsterdam, 1994.

Casdagli, M. C.: Recurrence plots revisited, Physica D, 108, 12-44, 1997.

Eckmann, J.-P., Kamphorst, S. O., and Ruelle, D.: Recurrence Plots of Dynamical Systems, Europhysics Letters, 5, 973-977, 1987.

Kantz, H. and Schreiber, T.: Nonlinear Time Series Analysis, University Press, Cambridge, 1997.

Martinson, D. G., Menke, W., and Stoffa, P.: An Inverse Approach to Signal Correlation, J. Geophys. Res. B6, 87, 4807-4818, 1982.

Nowaczyk, N. R., Frederichs, T. W., Kassens, H., NørgaardPedersen, N., Spielhagen, R. F., Stein, R., and Weiel, D.: Sedimentation rates in the Makarov Basin, Central Arctic Ocean - A paleo- and rock magnetic approach, Paleoceanography, 2001.

Prell, W. L., Imbrie, J., Martinson, D. G., Morley, J. J., Pisias, N. G., Shackleton, N. J., and Streeter, H. F.: Graphic Correlation of Oxygen Isotope Stratigraphy Application to the Late Quaternary, Paleoceanography, 1, 137-162, 1986.

Romano, M. C.: The Dark Side of Embedding, to be published.

Sakoe, H. and Chiba, S.: Dynamic programming algorithm optimization for spoken word recognition, IEEE Trans. Acoustics, Speech, and Signal Proc., 26, 43-49, 1978.

Takens, F.: Detecting Strange Attractors in Turbulence, pp. 366381, Springer, Berlin, 1981.

Thompson, R. and Clark, R. M.: Sequence slotting for stratigraphic correlation between cores: theory and practice, J. Paleolimnology, 2, 173-184, 1989.

Webber, Jr., C. L. and Zbilut, J. P.: Dynamical assessment of physiological systems and states using recurrence plot strategies, Journal of Applied Physiology, 76, 965-973, 1994.

Zbilut, J. P., Giuliani, A., and Webber, Jr., C. L.: Detecting deterministic signals in exceptionally noisy environments using cross-recurrence quantification, Physics Letters A, 246, 122$128,1998$. 